\title{
Therapeutic potential of pravastatin for random skin flaps necrosis: involvement of promoting angiogenesis and inhibiting apoptosis and oxidative stress
}

\author{
Jinti $\operatorname{Lin}^{1-3, *}$ \\ Chang Jia ${ }^{3,4, *}$ \\ Yongli Wang ${ }^{5}$ \\ Shanghong Jiang ${ }^{3}$ \\ Zhenyu Jia ${ }^{6}$ \\ Nan Chen ${ }^{3}$ \\ Shimin Sheng ${ }^{3}$ \\ Shihen $\mathrm{Li}^{1-3}$ \\ Liangfu Jiang ${ }^{1-3}$ \\ Huazi $X u^{1-3}$ \\ Kailiang Zhou ${ }^{1-3}$ \\ Yijie Chen ${ }^{3,7}$
}

'Department of Orthopaedics, The Second Affiliated Hospital and Yuying Children's Hospital of Wenzhou Medical University, Wenzhou 325027, People's Republic of China; ${ }^{2}$ Zhejiang Provincial Key Laboratory of Orthopaedics, Wenzhou 325027, People's Republic of China; ${ }^{3}$ The Second Clinical Medical College of Wenzhou Medical University, Wenzhou 325027, People's Republic of China; ${ }^{4}$ Pediatric Research Institute, The Second Affiliated Hospital and Yuying Children's Hospital of Wenzhou Medical University, Wenzhou 325027, People's Republic of China; ${ }^{5}$ Department of Orthopaedics, Huzhou Central Hospital, Huzhou 3/3300, People's Republic of China; ${ }^{6}$ School of Pharmaceutical Science, Wenzhou Medical University, Wenzhou, 325027, People's Republic of China; ${ }^{7}$ Department of Obstetrics and Gynecology, The Second Affliated Hospital and Yuying Children's Hospital of Wenzhou Medical University, Wenzhou 325027, People's Republic of China

*These authors contributed equally to this work

Correspondence: Kailiang Zhou

Department of Orthopaedics, The Second

Affiliated Hospital and Yuying Children's

Hospital of Wenzhou Medical University,

109 West Xueyuan Road, Wenzhou,

Zhejiang 325000, People's Republic of China

Tel +86577880028I5

Email zhoukailiang@wmu.edu.cn

Yijie Chen

Department of Obstetrics and Gynecology, The Second Affiliated Hospital and Yuying

Children's Hospital of Wenzhou Medical

University, 109 West Xueyuan Road,

Wenzhou, Zhejiang 325000, People's

Republic of China

Tel +86577880028I5

Email chirejoe@hotmail.com
This article was published in the following Dove Press journal:

Drug Design, Development and Therapy

Background: Random skin flap is frequently used in plastic and reconstructive surgery, but its distal part often occurs ischemia and necrosis. Pravastatin (Prava) with bioactivities of pro-angiogenesis, anti-apoptosis and anti-oxidative stress, may be beneficial for flap survival. Materials and methods: A modified McFarlane flap model was performed in SpragueDawley rats. The animals were divided into the Control and Prava groups and treated as follows: the Prava group was injected intraperitoneally with $2 \mathrm{mg} / \mathrm{kg}$ Prava for consecutive 7 days, and the Control group received an equal volume of vehicle daily. On day 7 , the necrosis skin flaps were observed, while visualization of blood flow below the tissue surface was performed by Laser Doppler blood flow imaging (LDBFI). Then animals were euthanized, and levels of angiogenesis, apoptosis and oxidative stress were analyzed.

Results: Prava decreased necrosis and edema of skin flaps compared with the Control group, with more blood flow in the flap under LDBFI. Prava treatment increased the mean vessels density, elevated the expression levels of angiogenic proteins (matrix metallopeptidase 9, vascular endothelial growth factor, Cadherin5) and antioxidant proteins (superoxide dismutase 1 (SOD1), endothelial nitric oxide synthase, heme oxygenase), and decreased the expression of apoptotic factors (BAX, CYC, Caspase3). In addition, malondialdehyde content was reduced, and glutathione level and SOD activity were increased in the skin flaps after treatment with Prava.

Conclusion: Prava promotes survival of random skin flap through induction of angiogenesis, and inhibition of apoptosis and oxidative stress.

Keywords: Pravastatin, random skin flap, angiogenesis, oxidative stress, apoptosis

\section{Introduction}

Postoperative tissue ischemia and necrosis in distal areas are crucial reasons for poor prognosis of random skin flaps. ${ }^{1,2}$ Random skin flap, where no specific blood vessel exists, mainly depends on new capillary network perfusion. ${ }^{3-5}$ It results in a flexibility of the skin flap due to no limitation of specific blood vessels, but also induces a vulnerability of tissue ischemia and necrosis. ${ }^{4}$ After the operation, angiogenesis begins from the pedicle of random skin flaps toward the distal part where neovascularization is easily inadequate. ${ }^{6}$ Therefore, tissue ischemia and necrosis usually occurs in the distal part, limiting the length-to-width ratio of random skin flaps (1.5-2.0:1). ${ }^{6}$ Thus, promoting angiogenesis can certainly increase random skin flap survival. However, as soon as angiogenesis and subsequent blood perfusion, ischemic reperfusion injury occurs. ${ }^{7,8}$ It means that oxidative stress 
occurs in the ischemia tissue and injuries random skin flaps, accompanied with cell apoptosis. ${ }^{9,10}$ Previous studies have demonstrated that promoting angiogenesis, and suppressing oxidative stress and apoptosis can enhance random skin flap survival.

Pravastatin (Prava) is a kind of statins which are 3-hydroxy-3-methyglutaryl-coenzume A (HMG-CoA) reductase inhibitors. ${ }^{11}$ Although statins are highly effective for lowering lipid level that have an established role in the prevention of coronary heart disease, the overall benefits of statins seem greater than the only lipid level lowering, suggesting their pleiotropic functions are beyond the inhibition of cholesterol biosynthesis. Importantly, the drugs are also commonly prescribed for the management of unstable angina pectoris and acute myocardial infarction, which are also ischemic diseases. ${ }^{11}$ As reported, statins are involved in endothelial function improvement, enhancement of atherosclerotic plaques stability, and attenuation of oxidative stress. ${ }^{12-14}$ In addition, current studies have demonstrated statins' efficacy on angiogenesis promotion and apoptosis inhibition. ${ }^{15-18}$ Several signaling cascades, as reported, have been implicated in these beneficial roles of statins. In endothelial cells, statins could exert the proangiogenic effect by activating the PI3K/Akt pathway, attenuate the oxidative stress via modulating ERK1/2 pathway, and inhibit apoptosis via a PPAR alpha-dependent pathway. ${ }^{12,15,16}$ However, it remains to elucidate whether Prava, or other statins, protects against ischemia and necrosis on random skin flaps.

In this study, we investigate the effect of Prava on random skin flaps survival and the involved mechanisms through determining the mean vessels density, the expression of angiogenic proteins (matrix metallopeptidase 9 [MMP9], vascular endothelial growth factor [VEGF], Cadherin5), antioxidant proteins (superoxide dismutase 1 [SOD1], endothelial nitric oxide synthase [eNOS], heme oxygenase [HO1]), and apoptotic factors (BAX, CYC, Caspase3). Furthermore, malondialdehyde (MDA) and glutathione (GSH) contents, and SOD activity were also analyzed.

\section{Material and methods}

\section{Animals and experimental model}

Adult male Sprague-Dawley rats (250-300 g) were acquired from Wenzhou Medical University (License No. SCXK [ZJ] 2005-0019). The animals were individually housed in a standard experimental cage in a controlled room (temperature: $23^{\circ} \mathrm{C} \pm 2^{\circ} \mathrm{C}$, humidity: $50 \% \pm 5 \%$, artificial lighting: 07:00-19:00 hrs), with food and water ad libitum. All experiments followed the Guide for the Care and Use of Laboratory Animals of the China National Institutes of Health, and were approved by the Animal Care and Use Committee of Wenzhou Medical University (wydw 2017-0022). The suffering of rats was minimized as much as possible.

Before surgery, animals were anesthetized by intraperitoneal injection of $2 \%(\mathrm{w} / \mathrm{v})$ pentobarbital sodium $40 \mathrm{mg} / \mathrm{kg}$. After that, the modified McFarlane flap model was performed in the rat dorsum (the same position in each rat): a caudal skin flap of $3 \times 9 \mathrm{~cm}$ was incised and separated from subcutaneous deep fascia on the back of each rat, while pedicle of the flap was preserved. ${ }^{19-21}$ Followed by that sacral arteries were sectioned. Finally, the flap was sutured to the donor bed by $4-0$ silk. The random skin flap area was equally divided into three parts from proximal part to distal part: area I, area II, area III.

\section{Animal groups and drug administration}

Forty-eight rats were randomly divided into the following two groups: the Control group and the Prava group ( $\mathrm{n}=24$ each). After the operation, each rat in the Prava group $(\mathrm{n}=24)$ was injected intraperitoneally with $2 \mathrm{mg} / \mathrm{kg}$ Prava for consecutive 7 days. ${ }^{22}$ Prava, provided by Sankyo (>99\%; Tokyo, Japan), was dissolved in $0.9 \%$ sodium chloride solution for a concentration of $0.5 \mathrm{mg} / \mathrm{mL}$. Rats in the Control group $(n=24)$ received an equal volume of vehicle daily for 7 days. Finally, animals were euthanized by an overdose of pentobarbital sodium and tissue samples were acquired and conserved.

\section{Experimental antibodies and reagents}

Antibodies were from the following companies: rabbit monoclonal anti-GAPDH (AP0063; Biogot Technology, Shanghai, People'sRepublic of China); rabbit monoclonal anti-Cadherin5 (A02632-2; Boster Biological Technology, Wuhan, People'sRepublic of China); rabbit monoclonal anti-SOD1, anti-MMP9, anti-HO1, anti-VEGF, anti-Caspase3 (CAPS3) $(12,452-1, \quad 19,003-1, \quad 10,375-2, \quad 10,269-1, \quad 10,701-1$; Proteintech Group, Chicago, IL, USA); rabbit monoclonal anti-endothelial anti-cytochrome $c(\mathrm{CYC})$, eNOS and antiBax (14,796, 11940S and 32,027; Cell Signaling Technology, Beverly, MA, USA); horseradish peroxidase (HRP)-conjugated IgG secondary antibody (Santa Cruz Biotechnology, Dallas, TX, USA). Pentobarbital sodium, DAB developer, and H\&E staining kit were acquired from 
Solarbio Science \& Technology (Beijing, People's Republic of China). SOD, GSH, and MDA assay kits were from Jiancheng Technology (Nanjing, People'sRepublic of China). The BCA Kit was purchased from Thermo Fisher Scientific (Rockford, IL, USA). The ECL Plus Reagent Kit was acquired from PerkinElmer Life Sciences (Waltham, MA, USA).

\section{Visual evaluation of flap survival}

During 7 days after operation, macroscopic development of random skin flaps was observed, including appearance, color, texture, hair condition of the flaps. Before rats were euthanatized on postoperative day 7 (POD 7), survival areas of the random skin flaps were measured by digital photographs of each rat's dorsum. Survival area was evidenced by pink and soft skin with new hair growth, whereas necrotic area was demonstrated by scabbing, hardening and dark nidus without new hair growth. Percentage of flap survival was calculated by ImageJ software (National Institutes of Health, Bethesda, MD, USA) according to the digital photographs:

\section{Percentage survival (\%) \\ $=$ extent of survival area/area of the whole flap $\times 100$.}

\section{Tissue edema evaluation of skin flap}

Tissue edema was evaluated according to the water content of the McFarlane flap, on POD 7 before the animals were euthanized. ${ }^{20,23}$ After euthanasia, six flap tissues per group were harvested and weighed (wet weight). Then, the flaps were dehydrated in an autoclave at $50^{\circ} \mathrm{C}$, and weighed daily until constant weight over 2 days (dry weight). The water content was calculated as:

$$
\begin{aligned}
& \text { Percentage water content }(\%) \\
& =([\text { wet weight }- \text { dry weight }] / \text { wet weight }) \times 100 .
\end{aligned}
$$

\section{Laser Doppler blood flow imaging (LDBFI) of the skin flap}

Before the rats were euthanized on POD 7, six rats per group were anesthetized and then the LDBFI was performed to assess blood supply of the McFarlane flap. The flap was scanned by Laserflo BPM (Vasamedic, St. Paul, MN, USA). LDBFI usually penetrate tissue surface deeply enabling enhanced visualization of small vessels below the skin flaps, perfect for angiogenesis evaluation. ${ }^{24}$ In the imaging, flap area with blood flow was marked by the LDBFI signal (green, yellow, and red, from weak to strong), whereas area lacking blood supply was marked by blue signal. The percentage of the blood flow area was calculated by ImageJ software (NIH, Bethesda, MD, USA):

Percentage of blood flow area $=$ area with LDBFI signal area (green, yellow, and red)/area of the whole flap $\times 100$.

\section{H\&E staining}

At POD 7, the animals were euthanized, and six samples $(0.5 \mathrm{~cm} \times 0.5 \mathrm{~cm})$ in the middle tissue of area II were harvested. Histologic examination was performed on these tissue samples, which were fixed in $4 \%$ neutral buffered formalin for $24 \mathrm{hrs}$, embedded in paraffin, sliced into $4 \mu \mathrm{m}$ thickness, and subsequently stained with H\&E staining (Solarbio Science \& Technology, Beijing, People'sRepublic of China). Under a light microscope (200× magnification), neovascularization, edema, and thickness of granulation tissue in flaps were evaluated. To assess angiogenesis level, mean vessel density (MVD) was counted manually by the number of microvessels per unit area $\left(/ \mathrm{mm}^{2}\right)$. Six random fields from three random sections of each tissue sample were determined for counting.

\section{Assays of MDA content, SOD activity, and GSH level}

To assess the oxidative stress levels of ischemic flaps, MDA, SOD, and GSH assays (Jiancheng Technology, Nanjing, People'sRepublic of China) were performed. ${ }^{25}$ On POD 7 , tissue samples $(0.5 \times 0.5 \mathrm{~cm})$ were obtained from area II flaps as above ( $\mathrm{n}=6$ per group). Then, the samples were weighed, homogenized, and diluted to $10 \%(\mathrm{v} / \mathrm{v})$ in ice bath. The homogenate was centrifuged at 3,500 rpm for 15 mins and the supernatant was harvested. Subsequently, MDA content was measured via reaction with thiobarbituric acid at 90 $100^{\circ} \mathrm{C}$, SOD activity was evaluated by the xanthine oxidase method, and GSH level was assessed using modified 5,5'dithiobis [2-nitrobenzoic acid] method.

\section{Immunohistochemistry (IHC)}

Sections from area II flaps ( $\mathrm{n}=6$ per group) were deparaffinized in xylene and rehydrated through a graded ethanol series. After washing, the sections were immersed in a 3\% hydrogen peroxide solution for $10 \mathrm{mins}$, put in $10 \mathrm{mM} / \mathrm{L}$ citrate buffer solution ( $\mathrm{pH} \mathrm{6.0)}$ for 20 mins $\left(95^{\circ} \mathrm{C}\right)$, and dipped in $10 \%(\mathrm{w} / \mathrm{v})$ BSA for 10 mins. Sections were incubated with monoclonal anti-CD34 (1:100), anti-VEGF (1:300), anti-caspase3 (1:200), anti-SOD1 (1:100), and 
anti-cadherin5 (1:100) antibodies overnight at $4^{\circ} \mathrm{C}$. After that, sections were incubated with HRP-conjugated secondary antibody for $1 \mathrm{hr}$, counterstained by hematoxylin, and visualized under $200 \times$ magnification using the DP2-TWAN image-acquisition system (Olympus Corp., Tokyo, Japan). To compare these protein expression and CD34-positive blood vessels, quantitative analysis was performed using Image-Pro Plus software (Media Cybernetics, Rockville, MD, USA). Six random fields of three random sections in each tissue sample were evaluated.

\section{TUNEL staining}

Terminal deoxynucleotide transferase-mediated (dUTP) nick-end labeling (TUNEL) staining was performed to detect the level of DNA fragmentation according to manufacturer's instructions. After deparaffinized, rehydrated, blocked, treated with $10.2 \mathrm{mM}$ sodium citrate buffer, and washed in PBS, sections were stained with in situ cell death detection kit (Roche China, Shanghai, People'sRepublic of China) for $30 \mathrm{mins}$ at $37^{\circ} \mathrm{C}$, and then were stained nuclei with DAPI. Finally, TUNEL positive cells were counted when six random fields of each slide were captured under a fluorescence microscope (Olympus Inc., Tokyo, Japan).

\section{Western blotting}

Tissue samples $(0.5 \times 0.5 \mathrm{~cm})$ as above $(\mathrm{n}=6)$ were acquired, homogenized, and the protein concentration was quantified using a BCA assay with BSA as the standard. Samples containing equal amounts of proteins $(60 \mu \mathrm{g})$ were separated in $12 \%(\mathrm{w} / \mathrm{v})$ gels and electro-transferred to polyvinylidene difluoride membranes. ${ }^{26,27}$ After blocked with 5\% skimmed milk, immunoblotting was probed with the following primary antibodies at $4^{\circ} \mathrm{C}$ overnight: Bax $(1: 1,000)$, CYC $(1: 1,000)$, CASP3 (1:1,000), MMP9 (1:1,000), VEGF $(1: 1,000)$, Cadherin5 (1:1,000), SOD1 (1:1,000), HO1 (1:1,000), eNOS $(1: 1,000)$, and GAPDH $(1: 1,000)$. After washing, the membranes were treated with HRP-conjugated secondary antibody $(1: 5,000)$ for $2 \mathrm{hrs}$ and visualized using the ECL Plus Reagent Kit. The band intensities were measured using Image Lab 3.0 software (Bio-Rad, Hercules, CA, USA).

\section{Statistical analyses}

Data were present as means \pm SEM. Statistical analysis was conducted using SPSS ver. 19 software (Chicago, IL, USA). Variance between the two groups was analyzed by the Student's $t$-test. Statistical significance was set at $p<0.05$, and $p<0.001$.

\section{Results}

\section{Prava elevated the survival of random}

\section{skin flaps}

After operation, the appearance of flaps turned to pale and swollen in each rat. At postoperative day 3 (POD 3), skin ischemic necrosis was observed in the distal portion of the flaps in both groups, as dark-colored, hard and contracted skin tissue without hair growth (Figure 1A). The necrosis almost occurred in area III flap, whereas area I and II flaps looked healthy. At POD 7, skin ischemic necrosis spread to area II flap in both groups. The necrosis in distal part of area II flap occurred as above described, whereas, in the caudal part of area II, the skin exhibited appearance as soft but superficially dark without hair growth, which was identified as survival but ischemic tissue. And area I flap still survived in both groups. After quantification, the Prava group significantly increased flap survival percentage compared with the Control group $(69.44 \pm 11.42 \%$ and 54.80 $\pm 11.30 \%$, respectively; $p=0.05$; Figure 1B). Furthermore, distal portion of subcutaneous tissue of flaps looked swollen and bruised with venous blood stasis, which was more obvious in the Control group (Figure 1C). After quantification, the water content of flaps was much lower in the Prava group (44.07 $\pm 6.62 \%)$ than that in the Control group (63.89 $\pm 7.42 \%$; $p=0.001$; Figure 1D). Under LDBFI (Figure 1E), the Prava group showed much higher percentage of blood flow area than the Control group $(67.75 \pm 10.17 \%$ and 32.51 $\pm 9.03 \%$, respectively; $p<0.001$; Figure $1 \mathrm{~F}$ ).

\section{Prava increased blood vessels in skin flaps}

As presented in Figure 2A, histological examination was conducted to reveal neovascularization in ischemic skin flap of the two groups. The MVD in the Prava group was $39.17 \pm 6.43 / \mathrm{mm}^{2}$, much higher than that in the Control group $\left(20.33 \pm 5.85 / \mathrm{mm}^{2} ; p<0.001\right.$; Figure $\left.2 \mathrm{C}\right)$. For further evaluation of MVDs in both groups, IHC for CD34 was performed to show endothelial cells in vessels (Figure 2B). After quantification, the number of CD34-positive vessels was significantly increased in the Prava group, compared with the Control group $\left(40.67 \pm 7.74 / \mathrm{mm}^{2}\right.$ vs $22.00 \pm 5.25$ / $\mathrm{mm}^{2} ; p<0.001$; Figure 2D).

\section{Prava promoted angiogenesis in skin flaps} IHC for the expression of VEGF and Cadherin5 was performed to assess angiogenesis level in both groups. VEGF and Cadherin5 were mostly found in vessels and stromal cells in area II flap (Figure $3 \mathrm{~A}$ and $\mathrm{C}$ ). 
Day 3

A

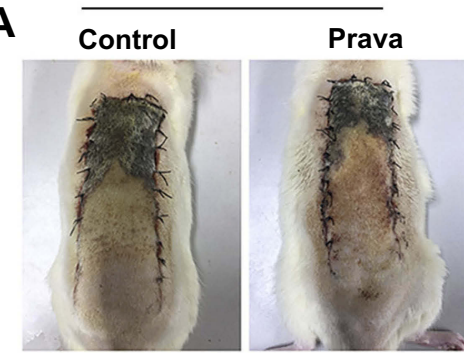

C

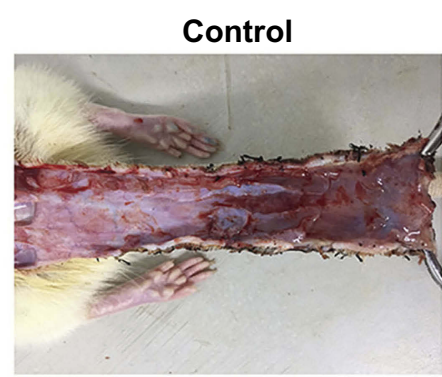

E

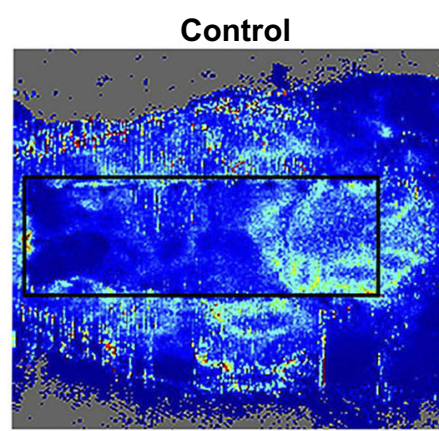

Day 7

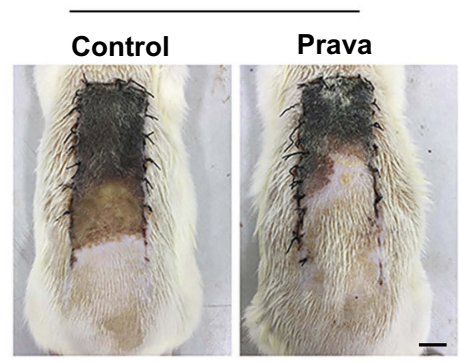

Prava

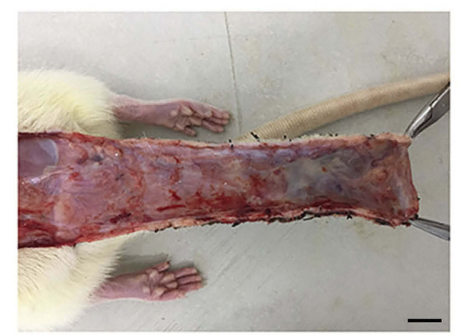

Prava

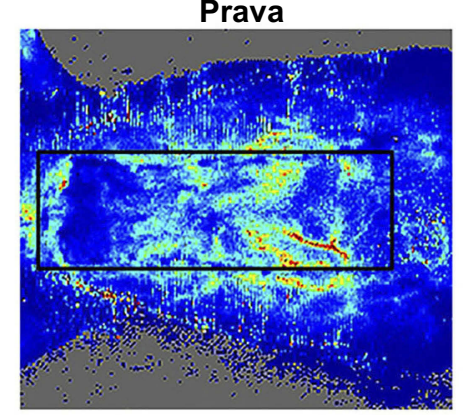

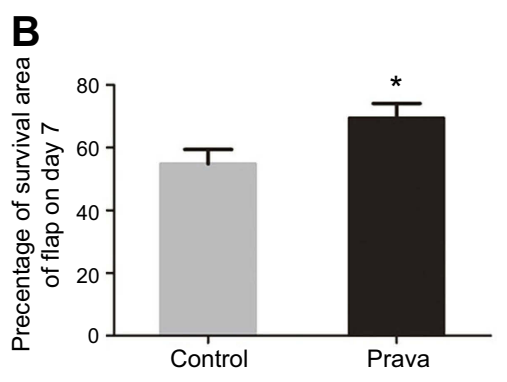

D

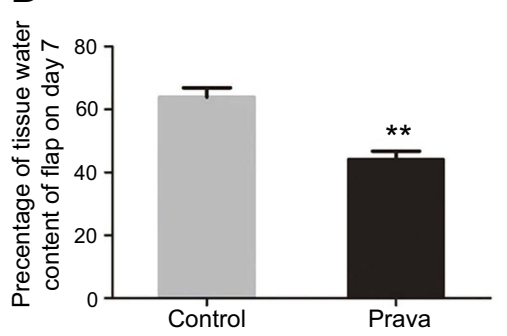

$0200400600 \quad 800$

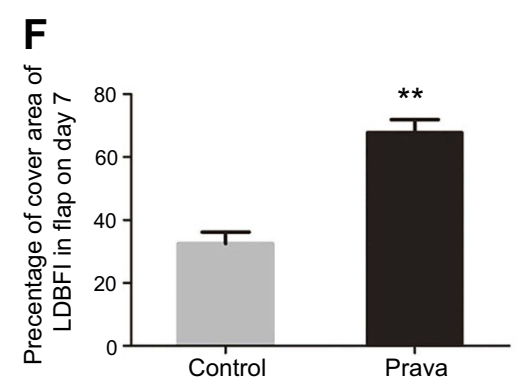

Figure I Prava elevated the survival of random skin flaps. (A) Digital photographs of macroscopic surface of postoperative flaps on POD 3 and 7 (scale bar, I cm). (B) Histogram about percentage survival of random skin flaps on POD 7. (C) Digital photographs of inner face of skin flaps, showing tissue edema and vessel statin of flaps. (D) Histogram about the percentage of tissue water content on POD 7. (E) Visualization of blood flow below the tissue surface measured by LDBFI. (F) Histogram of blood flow on POD 7. (Significance: $* p<0.05$ and $* * p<0.01$ vs the Control group. Data were expressed as mean \pm SEM. $n=6$ per group).

Abbreviations: Prava, pravastatin; POD 3, postoperative day 3; POD 7, postoperative day 7; LDBFI, Laser Doppler blood flow imaging; SEM, standard error of the mean.

Quantification analysis showed that Prava treatment significantly promoted VEGF expression compared with that in the Control group $(p<0.001$; Figure 3B). Similarly, Cadherin5 expression was also much higher in the Prava group $(p<0.001$; Figure 3D). Furthermore, levels of MMP9, VEGF, Cadherin5 in skin flaps were analyzed by Western blotting (Figure 3E-G). The result displayed that all the abovementioned proteins were upregulated in the Prava group than that in the Control group $(p=0.031$, $p=0.019, p=0.035$, respectively; Figure $3 \mathrm{H}-\mathrm{J}$ ).

\section{Prava attenuated apoptosis in skin flaps}

To examine cell apoptosis in the ischemic flaps, IHC for CASP3, as well as Western blotting for Bax, CYC, and
CASP3, was performed in both groups. As shown in IHC (Figure 4A), CASP3 was expressed in vascular endothelial and stromal cells, suggesting that apoptosis occurred, which might give an explanation of impairing vascular cells, decreasing blood flow supply, and eventually resulting in ischemia and necrosis of flaps. A remarkably decreased CASP3 expression was detected in the Prava group compared with the Control group ( $p<0.001$; Figure 4B). Moreover, the expression of Bax, CYC, and CASP3 after Prava treatment was analyzed by Western blotting (Figure 4C-E). Results exhibited that Prava treatment notably decreased the expression of $\mathrm{Bax}, \mathrm{CYC}$, and CASP 3 compared with the Control group ( $p=0.035, p=0.006$, $p<0.001$, respectively; Figure $4 \mathrm{~F}-\mathrm{H})$. In addition, TUNEL staining was conducted to evaluate the level of DNA 
A

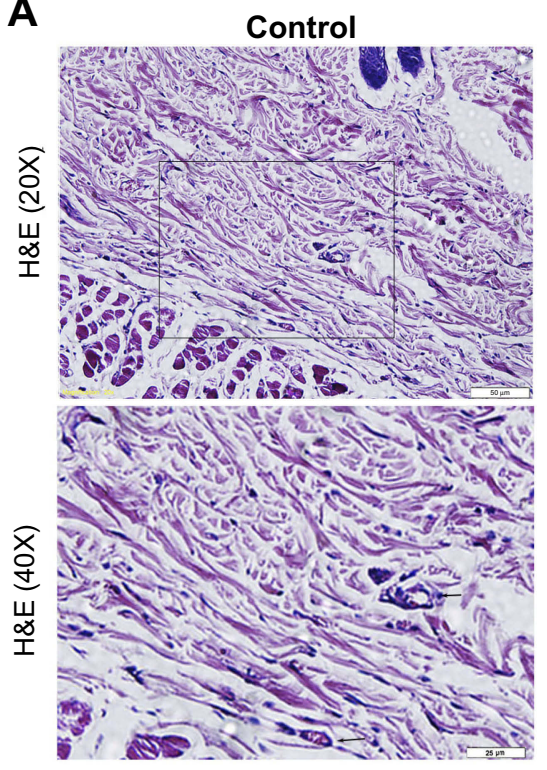

B

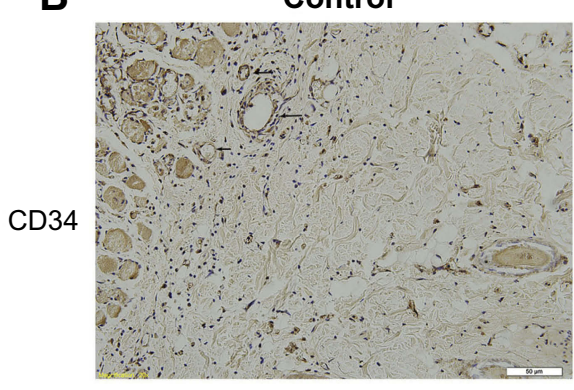

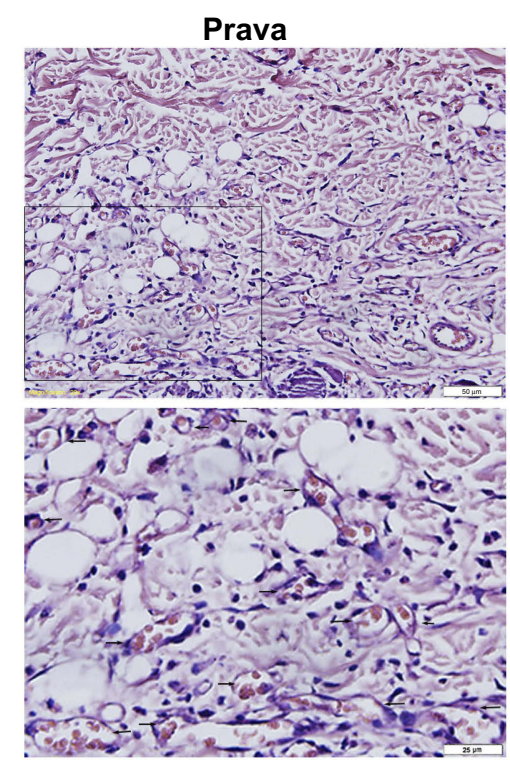

\section{Prava}
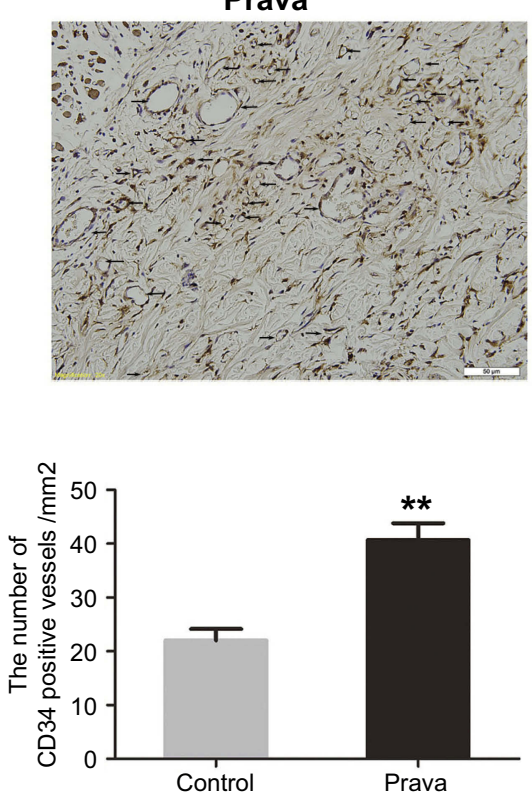

Figure 2 Prava increased blood vessels in skin flaps. (A) H\&E staining showing microvessels (located with black arrows) in ischemic skin flaps (area II) (original magnification $\times 200$ and $\times 400$; scan bar, 50 and $25 \mu \mathrm{m}$ ). (B) IHC of CD34-positive cells in flap area II (located with black arrows). (C) MVDs counted manually under H\&E staining. (D) CD34-positive vessel densities $\left(/ \mathrm{mm}^{2}\right)$ quantified under IHC. (Significance: ${ }^{*} p<0.05$ and ${ }^{* *} p<0.01$ vs the Control group. Data were expressed as mean $\pm S E M$. $n=6$ per group). Abbreviations: IHC, immunohistochemistry; MVDs, mean vessel densities; Prava, pravastatin; SEM, standard error of the mean.

fragmentation, in which the nuclei were stained blue and damaged DNA was green. The results showed that Prava group displayed lower levels of DNA damage $(p<0.001$, Figure 4I and J), suggesting that Prava treatment alleviated apoptosis in random skin flaps.

\section{Prava ameliorated the oxidative stress in skin flap}

Oxidative stress in skin flaps was evaluated by IHC for SOD1, Western blotting for SOD1, eNOS and HO1 expression, and content or activity analysis for SOD, GSH, and MDA. IHC for SOD1 expression was mainly performed in vascular endothelial and stromal cells in both groups (Figure 5A). After quantification, the integral absorbance of SOD1 expression in the dermis was increased in the Prava group compared with the Control group $(p<0.001$; Figure 5B). Western blotting for SOD1, eNOS, and HO1 showed that Prava treatment significantly increased SOD1, eNOS, and HO1 expression compared with the Control group $(p=0.008, p=0.003$, 
A
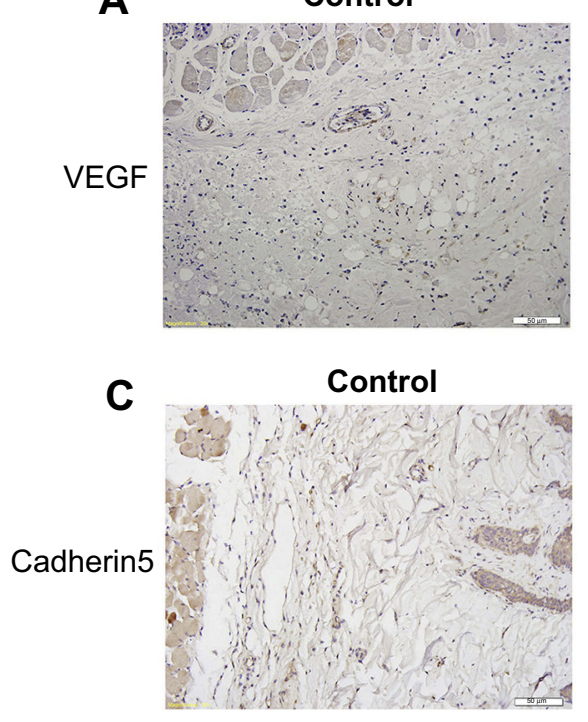

Prava

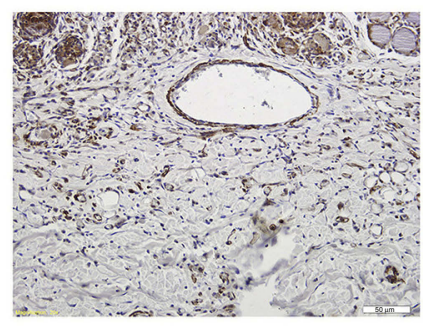

Prava

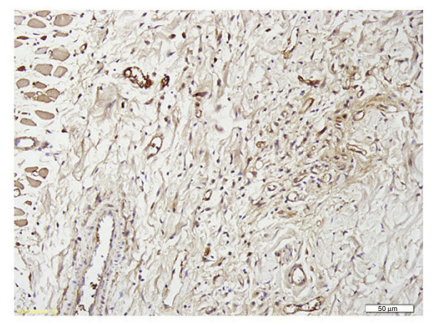

B

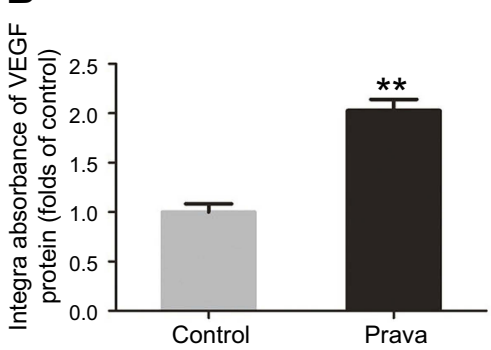

D

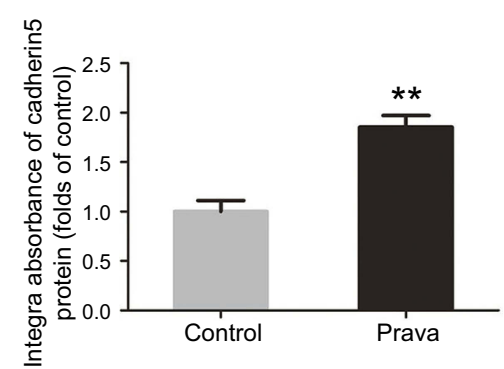

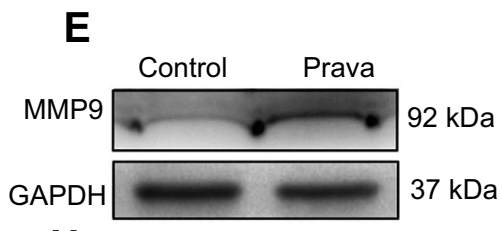

H

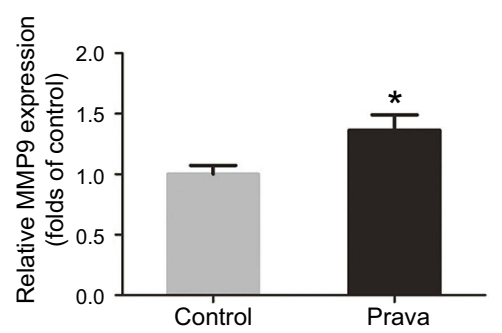

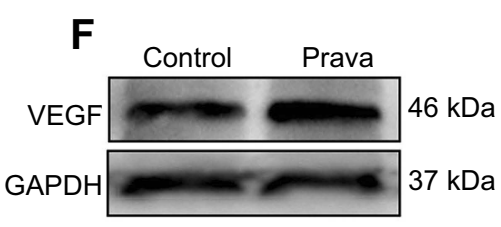

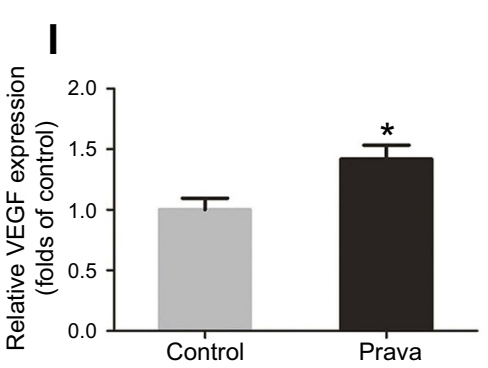

G G Control Prava
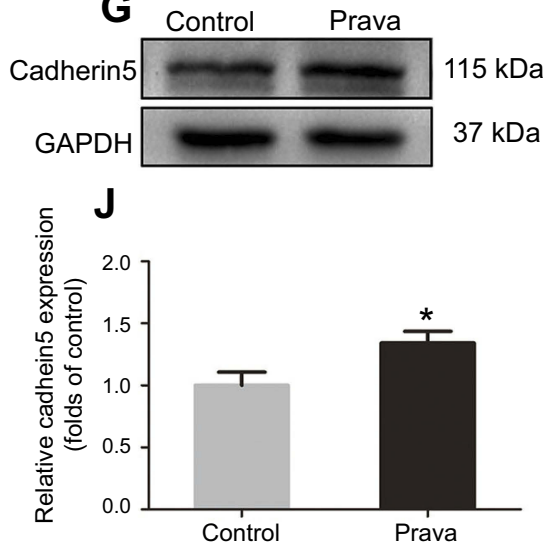

Figure 3 Prava promoted angiogenesis in skin flap. (A) IHC of VEGF expression in vessels and stromal cells in ischemic skin flaps (area II) (original magnification, $\times 200$; scan bar, $50 \mu \mathrm{m}$ ). (B) Histogram of integral absorbance of VEGF protein. (C) IHC of Cadherin5 expression in vascular endothelial and stromal cells in skin flaps (original magnification, $\times 200$; scan bar, $50 \mu \mathrm{m}$ ). (D) Histogram of integral absorbance of Cadherin5 protein. (E-G) Western blotting analysis of MMP9, VEGF, and Cadherin5 expression, with GAPDH as an internal control. (H-J) Histogram of optical density values of MMP9, VEGF, and Cadherin5 expression. (Significance: ${ }^{*}<<0.05$ and $* * * 0.01$ vs the Control group. Data were shown as means \pm SEM. $n=6$ per group).

Abbreviations: MMP9, matrix metallopeptidase 9; VEGF, vascular endothelial growth factor; IHC, immunohistochemistry; Prava, pravastatin; SEM, standard error of the mean.

$p=0.024$, respectively; Figure $5 \mathrm{C}-\mathrm{H})$. In addition, mean SOD activity was significantly increased in the Prava group $\left(60.00 \pm 12.00 \mathrm{U} \mathrm{mg}^{-1} \cdot\right.$ protein $\left.^{-1}\right)$ compared with the Control group $\left(41.83 \pm 11.89 \mathrm{U} \mathrm{mg}^{-1} \cdot\right.$ protein $^{-1}$; $p=0.025$; Figure 5I). The mean GSH level was remarkably elevated in the Prava group compared with that in the Control group $\left(2.32 \pm 0.44 \mathrm{nM} \mathrm{mg}{ }^{-1}\right.$ protein $^{-1}$ vs $1.50 \pm 0.26 \mathrm{nM} \mathrm{mg} \mathrm{mg}^{-1} \cdot \operatorname{protein}^{-1} ; p=0.003$; Figure 5J). Moreover, the mean MDA content in the Prava group was $38.00 \pm 6.82 \mathrm{nM} \mathrm{mg}^{-1} \cdot$ protein $^{-1}$ compared with $53.00 \pm 7.38 \mathrm{nM} \mathrm{mg}^{-1} \cdot$ protein $^{-1}$ in the Control group, with a significant difference ( $p=0.004$; Figure $5 \mathrm{~K}$ ).

\section{Discussion}

Based on this current study, Prava is demonstrated to be a novel therapeutic strategy for the management of ischemic random skin flap. When Prava was performed after the operation of skin flap, the agent treatment led to improved angiogenesis, reduced apoptosis, decreased oxidative stress, and increased random skin flap survival.

Ischemic necrosis in distal portion is a common and influential complication after random skin flap. ${ }^{1}$ The confluence of postoperative ischemia and subsequent ischemia reperfusion injury can cause necrosis of skin flaps, the 
A

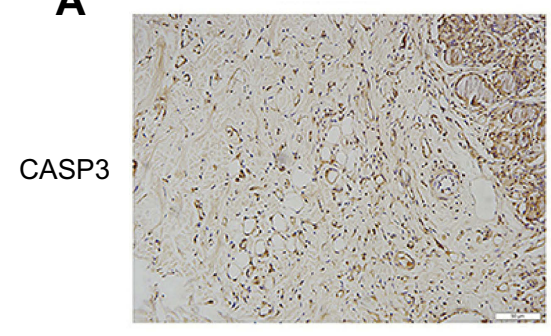

Prava

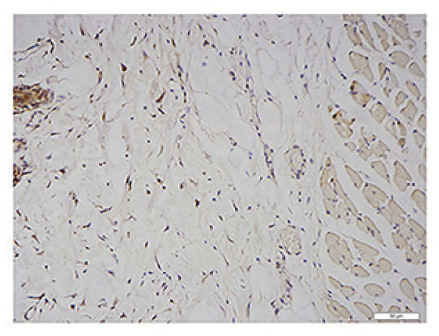

B

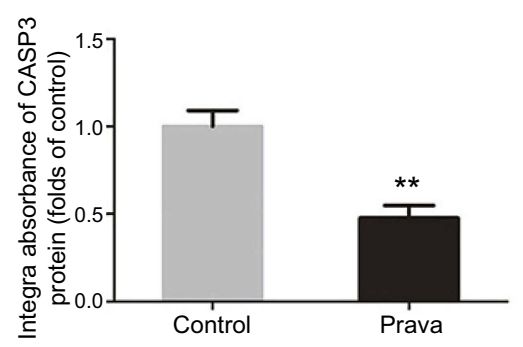

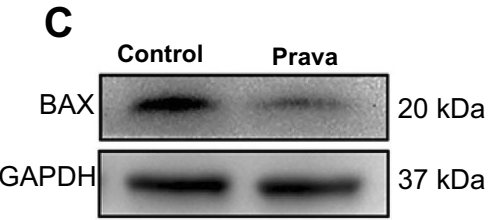

F

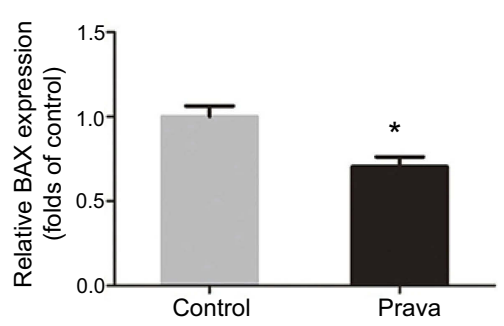

D

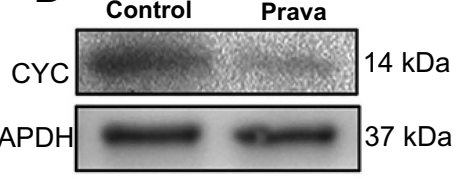

G

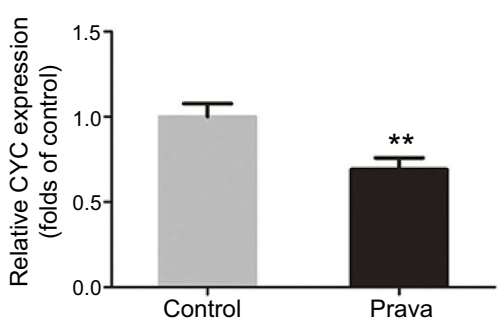

E

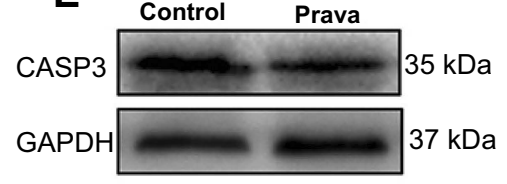

H

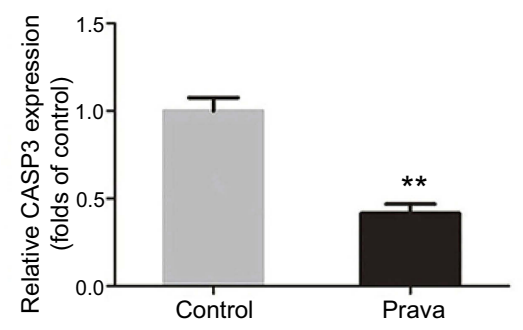

\section{I}
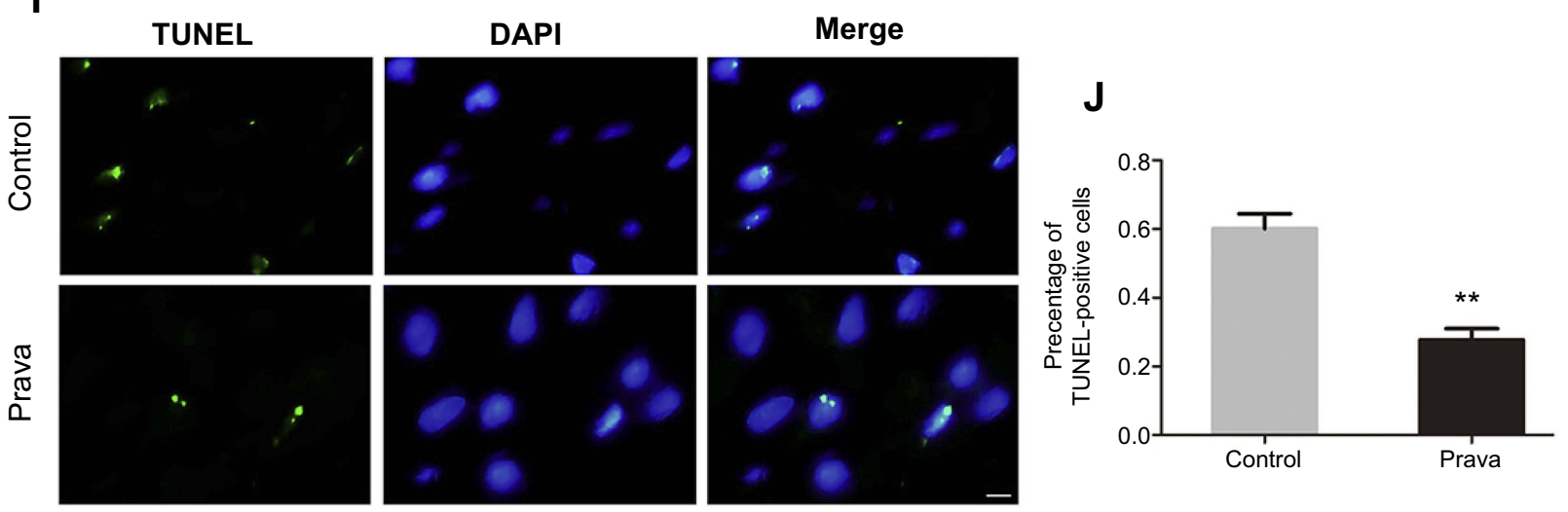

Figure 4 Prava attenuated cell apoptosis in ischemic skin flaps. (A) IHC of CASP3 mainly expressed in vascular endothelial and stromal cells in ischemic skin flaps (area II) (original magnification, $\times 200$; scan bar, $50 \mu \mathrm{m}$ ). (B) Histogram of integral absorbance of CASP3 protein under IHC. (C-E) Western blotting analysis for protein expression of Bax, CYC, and CASP3, with GAPDH as an internal control. (F-H) Histogram of optical density values of Bax, CYC, and CASP3 under Western blotting. (I) Immunofluorescence result of TUNEL staining showing the level of DNA damage in ischemic skin flaps. (J) Histogram showing the percentage of TUNEL-positive cells in two groups. (Significance: $* p<0.05$ and $*^{*} p<0.01$ vs the Control group. Data were presented as mean $\pm S E M$. $n=6$ per group).

Abbreviations: CYC, cytochrome c; CASP3, Caspase 3; IHC, immunohistochemistry; Prava, pravastatin; SEM, standard error of the mean.

pathology of which may stem from damage by free oxygen radials and cell apoptosis. ${ }^{28}$ Based on these reasons, agents with antioxidant, anti-apoptosis, and pro-angiogenesis might promote random skin flap survival. However, few studies have investigated the therapeutic functions of Prava for random skin flap. The current study demonstrated that Prava significantly decreased ischemic necrosis in skin flaps.
After the operation, skin flaps undergoes plasma exudation, congestion and edema, and ischemia. After these primary steps, the angiogenesis process mainly starts and brings new blood flow, ameliorating ischemia and necrosis of skin flaps. ${ }^{29}$ Prava has been reported to promote angiogenesis in endothelial cells by the activation of the PI3K/ Akt pathway. ${ }^{16}$ In the present study, H\&E staining and IHC 
A

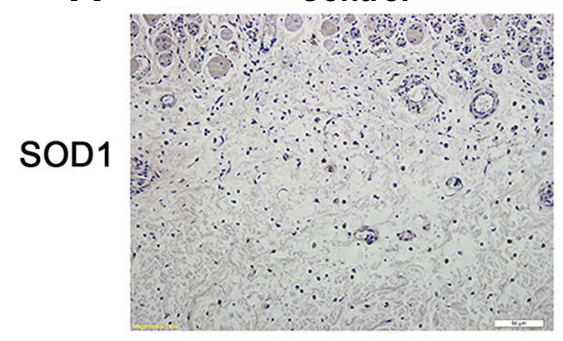

Prava

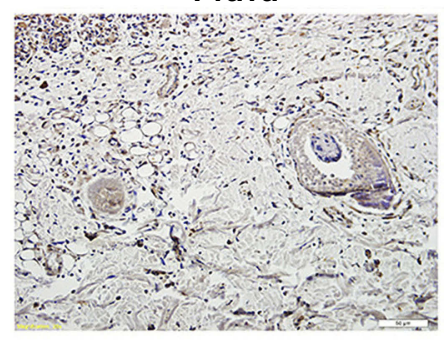

D

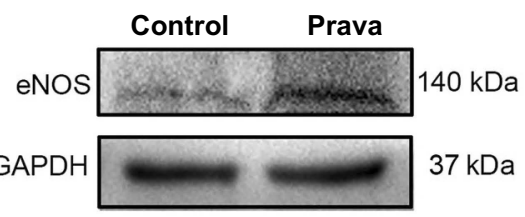

G

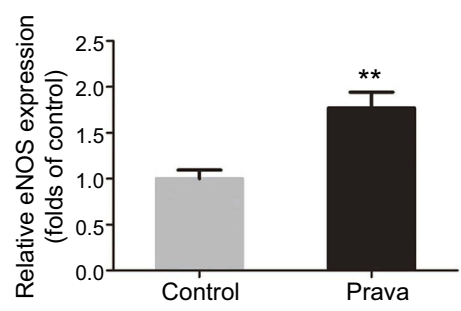

J

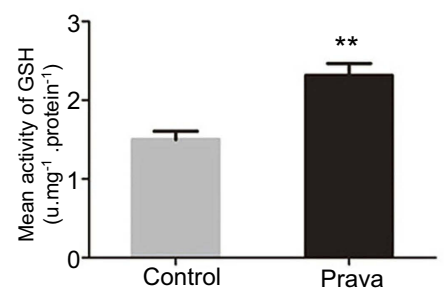

B

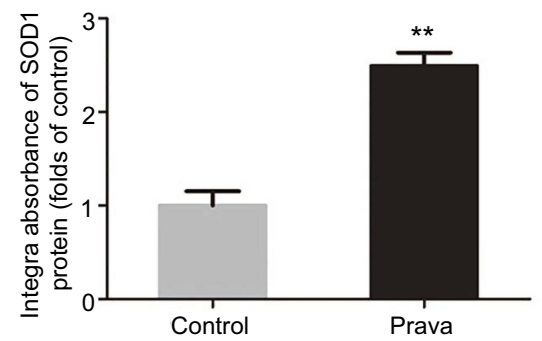

E

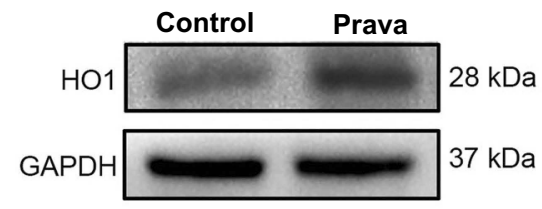

H

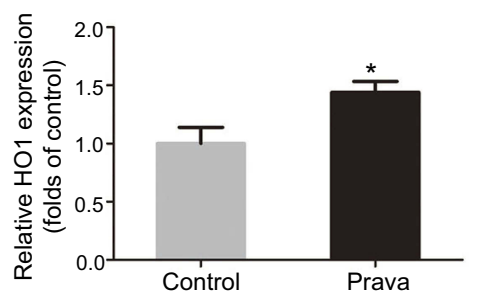

K

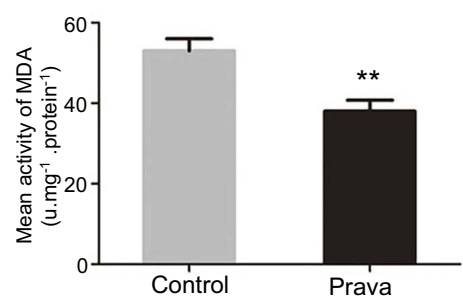

Figure 5 Prava ameliorated oxidative stress in ischemic skin flaps. (A) IHC for SODI mainly expressed in vessels and stromal cells in ischemic skin flaps (area II) (original magnification, $\times 200$; scan bar, $50 \mu \mathrm{m}$ ). (B) Histogram of integral absorbances of SODI expression under IHC. (C-E) Western blotting for protein expression of SODI, eNOS, and HOI, with GAPDH as an internal control. (F-H) Histogram of optical density values of SODI, eNOS, and HOI under Western blotting. (I) Histogram of SOD activities analyzed by the assay of the xanthine oxidase method. (J) Histogram of GSH levels analyzed via the assay of modified 5,5'-dithiobis method. (K) Histogram of MDA content analyzed via the modified TBA test. (Significance: ${ }^{*} p<0.05$ and ${ }^{*} p<0.0 \mathrm{I}$ vs the Control group. Data were expressed as mean $\pm S E M$. $n=6$ per group).

Abbreviations: SODI, superoxide dismutase I; eNOS, endothelial nitric oxide synthase; HOI, heme oxygenase; MDA, malondialdehyde; TBA, thiobarbituric acid; GSH, glutathione; IHC, immunohistochemistry; Prava, pravastatin; SEM, standard error of the mean.

of CD34 expression have shown higher MVD after Prava treatment. Consistent with higher blood flow according to LDBFI, we can hypothesize that Prava treatment improves vascularization in ischemic random skin flap and reduces necrosis. In the process of angiogenesis, MMP9 plays an essential role in the destruction of preexisting cell connections, VEGF is crucial proteins in mitosis of endothelial cells and Cadherin5 helps to form intercellular junctions which leads to maturation of new capillaries. ${ }^{30-32}$ Therefore, we evaluated the expression levels of the three proteins. IHC analysis indicated that Prava promoted the levels of VEGF and Cadherin5 in ischemic flaps. Moreover, Western blotting also shows higher expression of MMP9, VEGF, and Cadherin5 in the random skin flap with Prava management. In a word, Prava enhances angiogenesis and reduces necrosis in ischemic flaps with increased MMP9, VEGF, and Cadherin5.

It is obvious that skin flap survival is damaged by ischemic reperfusion injury after the operation. ${ }^{7}$ In the ischemic flaps, angiogenesis and following blood supply 
not only bring oxygen, but also result in the production of oxygen free radical, leading to damage of oxidative stress. ${ }^{33}$ In oxidative stress process, oxygen free radical acts on lipid peroxidation in cell membrane and invalidates proteins. ${ }^{34,35} \mathrm{MDA}$ is a reaction product of lipid peroxidation, which is proportional to the level of oxidative stress. ${ }^{36}$ At the same time, several antioxidant substances are synthesized and secreted to reduce oxidant stress during the process, including GSH and SOD. ${ }^{37}$ eNOS and HO1 are two enzymes with antioxidant activity. Yan et al had reported that statins could attenuate oxidative stress through regulating ERK1/2 pathway. ${ }^{12}$ In this study, we have also demonstrated that Prava can increase the expression of SOD1, eNOS, and $\mathrm{HO} 1$ in ischemic flaps according to IHC and Western blotting results. Moreover, the levels of GSH and SOD were elevated and MDA content was reduced in skin flaps with Prava treatment. All of these results suggest that Prava inhibits oxidative stress in random skin flaps.

Furthermore, administration of Prava might result in less apoptosis of endothelial cells and decreased permeability, leading to less edema. Consistent with our result, Chen et al found that Prava inhibited apoptosis through PPAR-alpha-dependent pathway in rat NRK-52E renal tubular epithelial cells. ${ }^{15}$ The apoptosis process was observed with impairment in vascular endothelial cells and basal layer. The damage of vascular endothelium might lead to plasmatic permeability, marked congestion and edema, ischemia and necrosis. ${ }^{29}$ In the process of apoptosis, the key step is mitochondrial swelling due to increased permeability of mitochondrial outer membranes, which is partly induced by Bax. ${ }^{38,39}$ Following that, CYC is released from the mitochondria, participating in the formation of apoptosome. ${ }^{39}$ Then CASP3 was activated through a cascade reaction, playing a role as an apoptosis executor which indicates the level of apoptosis in tissues. ${ }^{40}$ Thus, we evaluated the expression of Bax, CYC, CASP3 via IHC and Western blotting in the present study. And the result showed that Prava reduced apoptosis in random skin flaps. Therefore, we infer that Prava decreases apoptosis through inhibiting the expression of Bax, CYC, and CASP3, leading to less necrosis of random skin flaps.

There are some limitations in our study. The information from the investigation has not provided the assessment about the possibility of long-term effects of Prava. And optimal drug dose, timing, and duration of management also need to be investigated by well-designed prospective randomized controlled trials in the future. Nevertheless, evidence from current studies demonstrates that Prava is a simple, safe, effective, and economical management to decrease skin flap necrosis.

\section{Conclusion}

The management of Prava is a promising strategy to promote survival of random skin flaps. It exhibits the anti-necrotic property through inducing angiogenesis, and inhibiting oxidative stress and apoptosis.

\section{Consent for publication}

Hereby we declare that our institutes are aware of the work and declare consent for publication of the manuscript. We received consent to publish the animal experimental data from the animal ethical committee.

\section{Availability of data and materials}

All the data are presented in the paper.

\section{Acknowledgments}

This work was supported by grants from Natural Science Foundation of China (No. 81601705 to Kailiang Zhou, No. 81572227, No. 81873992 to Huazi Xu, No. 81701828 to Yijie Chen); Natural Science Foundation of Zhejiang Province (No. LQ18C010003 to Chang Jia, No. LY16H180008 to Yijie Chen); Zhejiang Provincial Medicine and Health Technology Project (No. 2017KY472 to Kailiang Zhou); Wenzhou Science and Technology Bureau Foundation (Y20150082 to Yijie Chen). Huzhou Science and Technology Bureau Foundation (2018GYB42 to Yongli Wang).

\section{Author contributions}

Jinti Lin and Chang Jia wrote the manuscript. Jinti Lin, Yongli Wang, and Shanghong Jiang prepared figures and collected samples. Zhenyu Jia, Nan Chen, Shimin Sheng, and Shihen Li analyzed data, Chang Jia, Kailiang Zhou, and Yijie Chen designed the experiments. Liangfu Jiang, Huazi $\mathrm{Xu}$, Kailiang Zhou, and Yijie Chen revised the manuscript. All authors contributed to data analysis, drafting and revising the article, gave final approval of the version to be published, and agreed to be accountable for all aspects of the work.

\section{Disclosure}

The authors report no conflicts of interest in this work. 


\section{References}

1. Karimipour M, Hassanzadeh M, Zirak Javanmard M, Farjah G. Oral administration of alanyl-glutamine and glutamine improve random pattern dorsal skin flap survival in rats. Iran J Basic Med Sci. 2018;21 (8):842-847. doi:10.22038/IJBMS.2018.29629.7153

2. Kwon JW, Hong SE, Kang SR, Park BY. Effect of human placental extract treatment on random-pattern skin flap survival in rats. $J$ Invest Surg. 2018;1-10. doi:10.1080/08941939.2017.1417518

3. Zhang P, Feng J, Liao Y, et al. Ischemic flap survival improvement by composition-selective fat grafting with novel adipose tissue derived product - stromal vascular fraction gel. Biochem Biophys Res Commun. 2018;495(3):2249-2256. doi:10.1016/j.bbrc.2017.11.196

4. Bai Y, Han YD, Yan XL, et al. Adipose mesenchymal stem cell-derived exosomes stimulated by hydrogen peroxide enhanced skin flap recovery in ischemia-reperfusion injury. Biochem Biophys Res Commun. 2018;500(2):310-317.

5. Hashimoto I, Abe Y, Ishida S, et al. Development of skin flaps for reconstructive surgery: random pattern flap to perforator flap. $J$ Med Invest. 2016;63(3-4):159-162.

6. Saito I, Hasegawa T, Ueha T, et al. Effect of local application of transcutaneous carbon dioxide on survival of random-pattern skin flaps. J Plast Reconstr Aesthet Surg. 2018;71(11):1644-1651.

7. Bai M, Liu Y, Yin D, et al. Inhibition of c-Jun N-terminal kinase signaling suppresses skin flap apoptosis in a rat ischemia and/or reperfusion model. $J$ Surg Res. 2016;206(2):337-346.

8. Pu CM, Liu CW, Liang CJ, et al. Adipose-derived stem cells protect skin flaps against ischemia/reperfusion injury via IL-6 expression. J Invest Dermatol. 2017;137(6):1353-1362.

9. Enzmann G, Kargaran S, Engelhardt B. Ischemia-reperfusion injury in stroke: impact of the brain barriers and brain immune privilege on neutrophil function. Ther Adv Neurol Disord. 2018;11:1756286418794184.

10. Lou Z, Wang AP, Duan XM, Hu GH, Zuo ML, Yang ZB. Role of ALK5/SMAD2/3 signaling in the regulation of NOX expression in cerebral ischemia/reperfusion injury. Exp Ther Med. 2018;16 (3):1671-1678. doi:10.3892/etm.2018.6377

11. Chan WW, Wong GT, Irwin MG. Perioperative statin therapy. Expert Opin Pharmacother. 2013;14(7):831-842. doi:10.1517/14656566.2013.782 003

12. Yan W, Li D, Zhou X. Pravastatin attenuates the action of the ETS domain-containing protein ELK1 to prevent atherosclerosis in apolipoprotein E-knockout mice via modulation of extracellular signal-regulated kinase 1/2 signal pathway. Clin Exp Pharmacol Physiol. 2017;44(3):344-352. doi:10.1111/1440-1681.12710

13. Blum A, Shamburek R. The pleiotropic effects of statins on endothelial function, vascular inflammation, immunomodulation and thrombogenesis. Atherosclerosis. 2009;203(2):325-330. doi:10.1016/j. atherosclerosis.2008.08.022

14. Lahera V, Goicoechea M, de Vinuesa SG, et al. Endothelial dysfunction, oxidative stress and inflammation in atherosclerosis: beneficial effects of statins. Curr Med Chem. 2007;14(2). doi:10.2174/092986707779313381

15. Chen HH, Chen TW, Lin H. Pravastatin attenuates carboplatin-induced nephrotoxicity in rodents via peroxisome proliferator-activated receptor alpha-regulated heme oxygenase-1. Mol Pharmacol. 2010;78(1):36-45. doi:10.1124/mol.109.061101

16. Liao Y, Zhang P, Yuan B, Li L, Bao S. Pravastatin protects against avascular necrosis of femoral head via autophagy. Front Physiol. 2018;9:307. doi:10.3389/fphys.2018.00307

17. Chen J, Zheng ZG, Li Y, et al. Statins induce angiogenesis, neurogenesis, and synaptogenesis after stroke. Ann Neurol. 2003;53 (6):743-751. doi:10.1002/ana.10555
18. Bergmann MW, Rechner C, Freund C, Baurand A, El Jamali A, Dietz R. Statins inhibit reoxygenation-induced cardiomyocyte apoptosis: role for glycogen synthase kinase $3 \beta$ and transcription factor $\beta$ catenin. J Mol Cell Cardiol. 2004;37(3):681-690. doi:10.1016/j. yjmcc.2004.05.025

19. Chen T, Tu Q, Cheng L, Li Z, Lin D. Effects of curculigoside A on random skin flap survival in rats. Eur $J$ Pharmacol. 2018;834:281-287. doi:10.1016/j.ejphar.2018.07.030

20. Lin R, Chen H, Callow D, et al. Multifaceted effects of astragaloside IV on promotion of random pattern skin flap survival in rats. $\mathrm{Am}$ $J$ Transl Res. 2017;9(9):4161-4172.

21. Mcfarlane RM, Heagy FC, Radin S, Aust JC, Wermuth RE. A study of the delay phenomenon in experimental pedicle flaps. Plast Reconstr Surg. 1965;35(35):245-262. doi:10.1097/00006534-196503000-00001

22. Orbay H, Hong H, Koch JM, Valdovinos HF, Hacker TA, Theuer CP; APA. Pravastatin stimulates angiogenesis in a murine hindlimb ischemia model a positron emission tomography imaging study with 64 Cu-NOTA-TRC105. Am J Transl Res. 2013;6(1):54.

23. Odland RM, Kizziar R, Rheuark D, Simental A. The effect of capillary ultrafiltration probes on skin flap edema. Otolaryngol Head Neck Surg. 2003;128(2):210-214. doi:10.1067/mhn.2003.69

24. Abraham A, Alabdali M, Alsulaiman A, et al. Laser Doppler flare imaging and quantitative thermal thresholds testing performance in small and mixed fiber neuropathies. PLoS One. 2016;11(11): e0165731. doi:10.1371/journal.pone.0165731

25. Lin R, Lin J, Li S, et al. Effects of the traditional Chinese medicine baicalein on the viability of random pattern skin flaps in rats. Drug Des Devel Ther. 2018;12:2267-2276.

26. Wang C, Mao C, Lou Y, et al. Monotropein promotes angiogenesis and inhibits oxidative stress-induced autophagy in endothelial progenitor cells to accelerate wound healing. J Cell Mol Med. 2017;22 (3):1583-1600. doi:10.1111/jcmm.13434

27. Zhou KL, Zhang YH, Lin DS, Tao XY, Xu HZ. Effects of calcitriol on random skin flap survival in rats. Sci Rep. 2016;6:18945. doi:10.1038/srep 18945

28. Kalapos MP. The tandem of free radicals and methylglyoxal. Chem Biol Interact. 2008;171(3):251-271. doi:10.1016/j.cbi.2007.11.009

29. Seyed Jafari SM, Shafighi M, Beltraminelli H, Geiser T, Hunger RE, Gazdhar A. Improvement of flap necrosis in a rat random skin flap model by in vivo electroporation-mediated HGF gene transfer. Plast Reconstr Surg. 2017;139(5):1116e-1127e. doi:10.1097/PRS.0000000000003259

30. Javerzat $S$, Franco $M$, Herbert $J$, et al. Correlating global gene regulation to angiogenesis in the developing chick extra-embryonic vascular system. PLoS One. 2009;4(11):e7856. doi:10.1371/journal. pone. 0007856

31. Townsend LE, Glover JL. Mitosis and angiogenesis in microwell endothelial cell culture. In Vitro Cell Dev Biol J Tissue Culture Assoc. 1991;27A(2):97-99. doi:10.1007/BF02630993

32. Park JK, Lee TW, Do EK, Moon HJ, Kim JH. Role of Notch1 in the arterial specification and angiogenic potential of mouse embryonic stem cell-derived endothelial cells. Stem Cell Res Ther. 2018;9 (1):197. doi:10.1186/s13287-018-0945-7

33. Koh YH, Park YS, Takahashi M, Suzuki K, Taniguchi N. Aldehyde reductase gene expression by lipid peroxidation end products, MDA and HNE. Free Radic Res. 2000;33(6):739-746.

34. Eldemerdash FM. Lipid peroxidation, oxidative stress and acetylcholinesterase in rat brain exposed to organophosphate and pyrethroid insecticides. Food Chem Toxicol. 2011;49(6):1346-1352.

35. Iqbal R, Akhtar MS, Hassan MQ, Jairajpuri Z, Akhtar M, Najmi AK. Pitavastatin ameliorates myocardial damage by preventing inflammation and collagen deposition via reduced free radical generation in isoproterenol-induced cardiomyopathy. Clin Exp Hypertens. Epub 2018 Sep 7. doi: 10.1080/ 10641963.2018.1501059 
36. Tsikas D. Assessment of lipid peroxidation by measuring malondialdehyde (MDA) and relatives in biological samples: analytical and biological challenges. Anal Biochem. 2016;524:13.

37. He F, Li J, Liu Z, Chuang CC, Yang W, Zuo L. Redox mechanism of reactive oxygen species in exercise. Front Physiol. 2016;7:486.

38. Estaquier J, Vallette F, Vayssiere JL, Mignotte B. The mitochondrial pathways of apoptosis. J Beijing Med Univ. 2002;942(1):157.
39. Gao W, Pu Y, Luo KQ, Chang DC. Temporal relationship between cytochrome c release and mitochondrial swelling during UV-induced apoptosis in living HeLa cells. $J$ Cell Sci. 2001;114(Pt 15):2855.

40. Porter AG, Jänicke RU. Emerging roles of caspase-3 in apoptosis. Cell Death Differ. 1999;6(2):99-104.

\section{Publish your work in this journal}

Drug Design, Development and Therapy is an international, peerreviewed open-access journal that spans the spectrum of drug design and development through to clinical applications. Clinical outcomes, patient safety, and programs for the development and effective, safe, and sustained use of medicines are a feature of the journal, which has also been accepted for indexing on PubMed Central. The manuscript management system is completely online and includes a very quick and fair peer-review system, which is all easy to use. Visit http://www. dovepress.com/testimonials.php to read real quotes from published authors. 